BMJ Open

Diabetes

Research

\& Care

\title{
Antidiabetic E4orf1 protein prevents hepatic steatosis and reduces markers of aging-related cellular damage in high fat fed older mice
}

\author{
Zahra Mostofinejad, ${ }^{1,2}$ Md Akheruzzaman (1) , ${ }^{1}$ Md Abu Bakkar Siddik (1) ," \\ Presheet Patkar, ${ }^{1}$ Nikhil V Dhurandhar, ${ }^{1}$ Vijay Hegde (D) ${ }^{1}$
}

\begin{abstract}
To cite: Mostofinejad Z, Akheruzzaman $\mathrm{M}$, Abu Bakkar Siddik M, et al. Antidiabetic E4orf1 protein prevents hepatic steatosis and reduces markers of aging-related cellular damage in high fat fed older mice. BMJ Open Diab Res Care 2021;9:e002096. doi:10.1136/ bmjdrc-2020-002096
\end{abstract}

ZM and MA contributed equally.

Components of this study have been presented at the American Society of Nutrition annual meeting in 2018 and at Obesity week 2020.

Received 24 December 2020 Revised 30 March 2021 Accepted 10 April 2021

Check for updates

(c) Author(s) (or their employer(s)) 2021. Re-use permitted under CC BY-NC. No commercial re-use. See rights and permissions. Published by BMJ.

'Department of Nutritional Sciences, Texas Tech University, Lubbock, Texas, USA

${ }^{2}$ Department of Nutrition and Environmental Toxicology, University of California, Davis, California, USA

Correspondence to Dr Vijay Hegde; vijay.hegde@ttu.edu

\section{ABSTRACT}

Introduction Older age is associated with greater prevalence of hyperinsulinemia, type 2 diabetes, and fatty liver disease. These metabolic conditions and aging are bidirectionally linked to mitochondrial dysfunction and telomere attrition. Although effectively addressing these conditions is important for influencing the health and the lifespan, it is particularly challenging in older age. We reported that E4orf1, a protein derived from human adenovirus Ad36, reduces hyperinsulinemia, improves glucose clearance, and protects against hepatic steatosis in younger mice exposed to high fat diet (HFD). Here, we tested if E4orf1 will improve glycemic control, liver fat accumulation, mitochondrial integrity, and reduce telomere attrition in older mice.

Research design and methods We used 9-month-old mice that inducibly expressed E4orf1 in adipose tissue and non-E4orf1 expressing control mice. Mice were maintained on a $60 \%$ (kcal) HFD for 20 weeks and glycemic control was determined by intraperitoneal glucose tolerance test at week 20. Following 20 weeks of HF-feeding, mice were sacrificed and liver tissues collected to determine the expression of aging genes using qRT-PCR based $\mathrm{RT}^{2}$ Profiler PCR array.

Results Compared with the control mice, E4orf1 significantly improved glycemic control and reduced hepatic steatosis and fibrosis. Additionally, E4orf1 maintained markers of mitochondrial integrity and telomere attrition.

Conclusion E4orf1 has the potential to improve glycemic control in older mice, and the improvement persists even after longer term exposure. E4orf1 expression also maintains mitochondrial integrity and telomere attrition, thus delaying age-associated diseases. This provides strong evidence for therapeutic utility of E4orf1 in improving age-associated metabolic and cellular changes that occur with aging in humans

\section{INTRODUCTION}

Aging is a very intricate process that leads to the structural and functional decline of organs and tissues, marked by chronic systemic inflammation, oxidative stress, DNA damage, impaired mitochondrial function, cellular senescence, and tissue dysfunction. ${ }^{1}$ Aging

\section{Significance of this study}

What is already known about this subject?

- Metabolic disorders such as hyperinsulinemia, type 2 diabetes and fatty liver disease and aging are bidirectionally associated with mitochondrial dysfunction and telomere attrition.

- Modifying certain biomarkers like deregulated nutrient sensing, mitochondrial dysfunction, cellular senescence caused by telomere attrition and genomic instability are strategies to improve the health span of an individual.

- Despite lifestyle modifications and antidiabetic agents showing health benefits, there are limitations and better therapeutic interventions are needed for delaying aging-related risk factors.

What are the new findings?

- In high fat fed older mice, adenoviral protein E4orf1 significantly improves glycemic control in an insulin independent manner.

- E4orf1 expression reduces hepatic steatosis and fibrosis despite high fat diet in these mice.

- E4orf1 expression also reduces aging-related hepatic markers of mitochondrial integrity and telomere attrition.

How might these results change the focus of research or clinical practice?

- Given the challenges among the elderly in improving aging associated comorbidities, use of E4orf1 as a therapeutic target in improving glycemic control, protecting against hepatic fat accumulation and reducing aging-associated cellular damage despite chronic high fat diet will be clinically desirable.

shares many chronic metabolic disorders with obesity and type 2 diabetes (T2D), such as insulin resistance, poor glycemic control, and chronic low-grade inflammation. ${ }^{2}$ Advanced age is also associated with ectopic lipid accumulation in the liver and can increase the prevalence of nonalcoholic fatty liver disease (NAFLD). ${ }^{3}$ While the contribution of obesity, 
T2D, NAFLD, and nonalcoholic steatohepatitis (NASH) in the etiology of aging is somewhat ambiguous, given the complexity, the onset of these diseases through metabolic alterations and acceleration of aging could be a vicious cycle. ${ }^{4}$

One of the key factors related with cellular aging is telomere attrition and damage. ${ }^{5}$ Several studies have shown that patients with T2D have shorter telomeres in white blood cell and in pancreatic $\beta$-cells than non-T2D individuals. ${ }^{67}$ Additionally, decline in mitochondrial function or an increase in reactive oxygen species (ROS) has also been implicated in aging. ${ }^{8}$ Further, it has been shown that telomere dysfunction is associated with a decline in mitochondrial function. ${ }^{9}$ Alternatively, hyperglycemia has been shown to induce mitochondrial dysfunction, ${ }^{10}$ suggesting a bidirectional relationship between T2D and aging associated telomere attrition and mitochondrial dysfunction.

In view of the complex nature of the aging process, quantitative biomarkers like epigenetic alterations, deregulated nutrient sensing, mitochondrial dysfunction, cellular senescence caused by telomere attrition and genomic instability predict health span of an individual ${ }^{11}$ and potentially can be modified. Dietary restriction and exercise have been the most studied interventions in improving aging associated risk factors. ${ }^{12}{ }^{13}$ However, poor compliance associated with these lifestyle modifications makes them challenging, especially for the elderly. Well maintained glycemic control could prevent or delay the development of multiple age-related disorders and might slow the aging process. ${ }^{14}$ Currently, several antidiabetic agents are used for maintaining glycemic control. Common among them is insulin sensitizer metformin, which also improves health span in mice ${ }^{15}$ and shows reduced mortality in patients with diabetes compared with patients with diabetes not receiving metformin. ${ }^{16}$ One of the limitations with efficacy of many antidiabetic drugs is the requirement of a functional insulin signaling pathway, which is often impaired during T2D. Therefore, therapeutic interventions for delaying aging related risk factors or for a healthy aging should improve metabolic impairments in glycemic control and hepatic metabolic dysfunctions despite inability to sustain lifestyle modifications among the elderly.

We have previously shown that the E4orf1 protein, derived from human adenovirus Ad36, improves glucose clearance in an insulin-independent manner in chow and high fat diet (HFD) fed 6-10 weeks old mice. ${ }^{17-19}$ In a recent study, adipose tissue-specific expression of E4orf1 in a transgenic mouse model reduced endogenous insulin requirement and reduced lipid synthesis in liver and adipose tissue. ${ }^{17}$ There are no reports of the effect of E4orfl in humans. However, the glycemic and hepatic effects of E4orfl were recognized through similar effects of adenovirus 36, the virus from which E4orf1 is derived. Experimental Ad36 infection of rodents improves glycemic control and reduces hepatic steatosis. ${ }^{20} 21$ Cross-sectional and longitudinal studies showed that humans who are naturally exposed to Ad36 have better glycemic control and, importantly, lower hepatic steatosis or NAFLD prevalence. ${ }^{22}{ }^{23}$ All these features of E4orf1 protein appear to be attractive for the alleviation of several risk factors of aging, which may include improved glycemic control and protection against NAFLD but needs further investigation, particularly in an older mouse model.

In the current study, we determined if E4orfl can improve glycemic control in an insulin independent manner in HFD-fed older mice. More specifically, we wanted to test if E4orf1 mediated protection against hepatic steatosis can potentially prevent its progression to fibrosis and delay aging-associated molecular dynamics.

\section{RESEARCH DESIGN AND METHODS}

\section{Animal model and experimental design}

Transgenic C57BL/6J mice expressing Ad36E4orf1 protein $(\mathrm{n}=7, \mathrm{M}=4, \mathrm{~F}=3)$ or non-transgenic littermates (C57BL/6 mice, $\mathrm{n}=8, \mathrm{M}=4, \mathrm{~F}=4$ ) from our breeding colony were included in this study. Animals were handled following Texas Tech University Institutional Animal Care and Use Committee guidelines. All mice were group-housed, on a 12 hours light/12 hours dark cycle at $25^{\circ} \mathrm{C}$, with ad libitum access to chow diet (protein $24 \%$, carbohydrate $60 \%$, and fat $16 \%$ ) and water. Weekly body weight was recorded. After taking baseline measurements at 9 months of age, animals were switched to a high fatdoxycycline diet (HF-dox; 60\% kcal from fat, dox 600 $\mathrm{ppm} / \mathrm{kg}$ ) until their sacrifice (at week 20). Following 20 weeks of HF-dox feeding, blood glucose was measured after 4 hours fasting and $15 \mathrm{~min}$ after a glucose challenge as described previously. ${ }^{17}$ Serum insulin was also assessed by ELISA (EMD Millipore, Cat. EZRMI-13K). Mice were then sacrificed using $\mathrm{CO}_{2}$ asphyxiation and cervical dislocation and cardiac puncture blood was collected. Liver and other peripheral tissues were harvested, frozen immediately in liquid nitrogen, and preserved at $-80^{\circ} \mathrm{C}$ for biochemical analysis.

\section{Western blotting}

Liver, skeletal muscle, and adipose tissue depots were homogenized in radioimmune precipitation assay (Cell Signaling Cat. 9806) buffer with added protease inhibitor. Thereafter, quantification of the extracted protein was performed by bicinchoninic acid (BCA) protein assay (Thermo Scientific Pierce BCA Protein Assay Kit, Cat. 23225). Protein $(30 \mu \mathrm{g})$ from each sample was separated by sodium dodecyl sulfate-polyacrylamide gel electrophoresis, transferred on to a polyvinylidene difluoride membrane, blocked for 1 hour with $5 \%$ non-fat milk in tris-buffered saline and tween followed by overnight incubation with primary antibody. Afterward, the membrane was immunoblotted with appropriate secondary antibody conjugated with horseradish peroxidase (1:4000) and treated with clarity western ECL substrate (Bio-Rad, Cat. 170-5061) reagent. All protein bands were normalized 
by calculating the ratio of band intensity to respective housekeeping GADPH protein bands. ChemiDoc Imaging System (Biorad) and ImageJ software were used for visualization and quantification of protein bands, respectively. Antibodies used in this study were as follows: E4orf1 (Custom made, Protein Tech), TNF- $\alpha$ (Cell Signaling, Cat. 3707S), pAkt (S473) (Cell Signaling, Cat. 9271L), total Akt (Cell Signaling, Cat. 4691S), Ras (Cell Signaling, Cat. 3965S), GLUT2 (Santa Cruz Biotechnology, GLUT4 (R\&D Systems, Cat. MAB1262), PPAR- $\gamma$ (Santa Cruz Biotechnology, Cat. sc-7273), Cat. sc-9117), and GAPDH (Cell Signaling, Cat. 2118S).

\section{Real-time quantitative PCR (RT-qPCR)}

Liver RNA was extracted using the RNeasy Plus Universal Mini Kit (Qiagen, Cat. 73404). Reverse transcription was performed using the Maxima cDNA Synthesis Kit (Thermo Fisher Scientific, Cat. K1681). Generated cDNA was amplified using SsoAdvanced Universal SYBR Green Supermix (Bio-Rad, Cat. 172-5271) in a BioRad CFX RT-PCR detection system. The final volume of RT-qPCR reaction mix was $20 \mu \mathrm{L}$ that contains $25 \mathrm{ng}$ of cDNA $(5 \mu \mathrm{L}), 450 \mathrm{nM}$ of the forward and reverse primers $(1.8 \mu \mathrm{L}), 1 \mathrm{X}$ SYBR Green $(10 \mu \mathrm{L})$, and water $(3.2 \mu \mathrm{L})$. We randomly selected $\mathrm{WT}(\mathrm{n}=5)$ and $\mathrm{E} 4(\mathrm{n}=4)$ mice as biological replicates for RT-qPCR analysis. Duplicates $(\mathrm{n}=2$ technical replicates) were run for all the samples and average Ct values were calculated. The relative mRNA expression was determined using the $2^{\wedge}(-\Delta \Delta \mathrm{Ct})$ method while the Tbp1 or beta-2 microglobulin (B2m) gene was used as a reference gene. Gene primer list and sequences are as described previously. ${ }^{17}$

\section{Histopathology}

Histological analysis of the liver samples was carried out by IDEXX BioAnalytics (Columbia, MO) as described previously. ${ }^{17}$ Briefly, liver sections from randomly selected five mice were stained with hematoxylin and eosin (H\&E) following fixation for 24 hours in formalin. Picrosirius red was used to stain liver sections for visualizing liver fibrosis. A pathologist blinded to treatment group identity performed the microscopic scoring. Observed microscopic changes were graded using rodent NAFLD grading system ${ }^{24}$ with one modification; evaluation of 10 fields, as opposed to 5 fields were used to count the number of inflammatory foci. Finally, a standard grading system was employed to get the fibrosis score: $0=$ no significant fibrosis; $1=$ mild fibrosis; $2=$ moderate fibrosis; and $3=$ massive fibrosis.

\section{RT-PCR array for aging-related genes}

An RT² Profiler PCR Array (Qiazen, Cat. no. PAMM-178Z) was performed to assess the expression of genes involved in aging-related pathways in the liver. Liver cDNA ( $25 \mathrm{ng}$, see previously described method "Real-time quantitative PCR") was used in the real-time RT ${ }^{2}$ Profiler PCR Array in combination with RT ${ }^{2}$ SYBR Green qPCR Mastermix (Qiazen, Cat. no. 330529) in Bio-Rad CFX RT-PCR detection system. The assay profiled 84 aging-related genes, 5 reference genes, and 7 control genes. Ct values were exported to an Excel file to create the table of $\mathrm{Ct}$ values followed by uploading at http:/ /www.qiagen.com/ geneglobe for data analysis. Four samples, randomly chosen from control and E4orf1 groups, were assigned to control and test group during analysis. Ct values were normalized by the geometric mean of a full panel of reference genes (Gapdh, Hsp90ab1, and Actb) based on an automatic selection. Geneglobe then calculated fold changes using $2^{\wedge}(-\Delta \Delta \mathrm{Ct})$ method. The protein-protein interaction of differentially expressed gene's products was analyzed and visualized by the STRING 11.0 (https:/ / string-db.org/) and Cytoscape 3.8.0, respectively.

\section{Statistical analysis}

All results are presented as mean $\pm \mathrm{SE}$ of the mean. All mean values were compared between control and E4orf1 group using Welch's $t$ test assuming unequal variance.

\section{RESULTS}

E4orf1 improves glycemic control in older mice following long-term HF exposure

Previous studies examined the effects of inducibleadipose tissue-specific transgenic expression of E4orf1 in younger mice (6-12 weeks old) that were on $60 \%$ HFD for 6 weeks. ${ }^{18}$ To test the effect of E4orf1 in older mice that were on HFD for a longer duration, 9-month-old transgenic E4orf1 $(n=7)$ and non-transgenic control $(n=8)$ mice were maintained on a HFD containing doxycycline for 20 weeks to induce hyperglycemia. As expected, the protein expression analysis showed doxycycline-induced E4orf1 transgene expression in the E4orf1 mice group, but not in the control group (data not shown). There was no significant difference in weekly body weight between the control and E4orfl mice over the 20 weeks of HFDfeeding (figure 1A).

Glycemic control was determined by intraperitoneal glucose challenge at week 20. E4orf1 mice displayed significantly lower glucose $(\mathrm{p}<0.04$; figure $1 \mathrm{~B})$ at $\mathrm{t}=15$ and lower percent increase from baseline $(\mathrm{p}<0.03$; figure $1 \mathrm{C})$ compared with control mice. E4orf1 expression also significantly improved the product of glucose and insulin at $\mathrm{t}=15$ in E4orf1 mice $(\mathrm{p}<0.03$; figure 1D) compared with control mice. This indicated lower requirement of endogenous insulin for glucose disposal.

To determine serum metabolites and liver outcomes, we measured hepatic triglycerides, liver weight (\% of body weight) and serum free fatty acids (FFA). We did not observe any significant difference between E4orf1 expressing mice compared with control mice (figure E-G).

\section{E4orf1 improves glycemic control by activating the distal insulin Ras/PI3K pathway}

To determine the tissue-specific molecular changes associated with systemic improvement in glycemic control, we examined the protein expression of Ras, Akt and 
A.

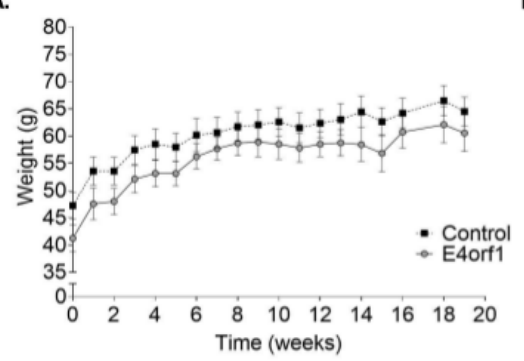

D.

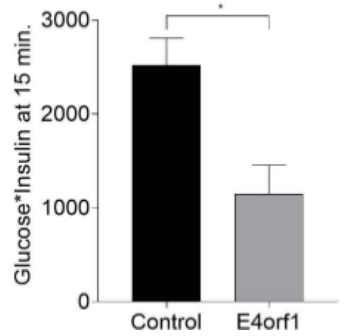

B.

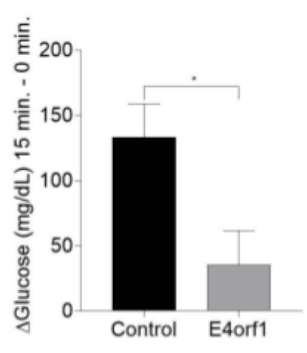

E.

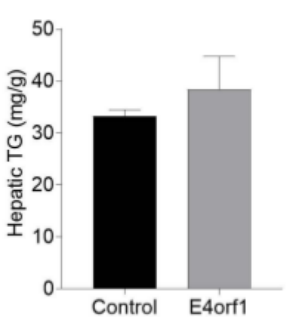

F.

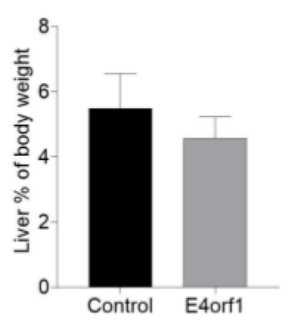

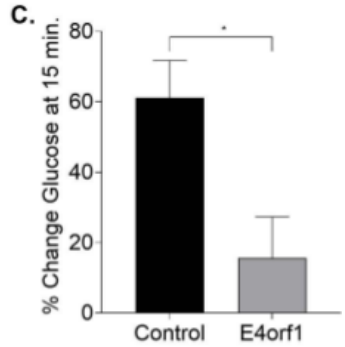

G.

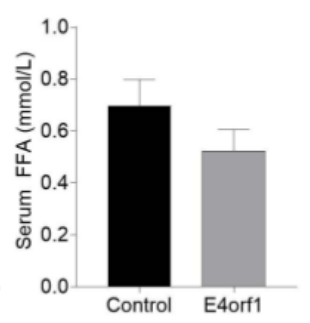

Figure 1 E4orf1 improves glycemic control in older mice. (A) Weekly body weight measurement does not show any difference between control and E4orf1 mice. (B) E4orf1 expression in mice significantly reduces glucose peak following a glucose load measured as delta change in glucose levels between 0 vs $15 \mathrm{~min}$ and (C) displays significantly lower per cent increase in glucose from 0 to $15 \mathrm{~min}$. (D) E4orf1 expression also significantly improves glycemic control determined as a product of blood glucose and serum insulin. (E) Hepatic triglyceride $(\mathrm{mg} / \mathrm{g})$ level in control and E4orf1 mice. (F) Liver per cent of body weight in control and E4orf1 mice. (G) Serum FFA (mmol/L) level in control and E4orf1 mice. Welch's $t$-test: ${ }^{*} p<0.05$. FFA, free fatty acids; TG, triglyceride.

Glut4 genes involved in the distal insulin signaling Ras/ PI3K pathway. E4orf1 expressing transgenic mice showed significantly increased phosphorylation of Akt and increased protein abundance of Glut 4 in the retroperitoneal, epididymal and inguinal fat depots compared with non-transgenic control mice (figure 2A-F). However, we did not observe any difference in protein expression for Ras between the two groups of mice (figure 2A-F). In the skeletal muscle, E4orf1 expressing mice showed significantly increased protein expression for Ras and pAkt compared with control mice (figure $2 \mathrm{G}, \mathrm{H}$ ). In the liver, there was no difference for TNF- $\alpha$, GLUT2, and PPAR- $\gamma$ protein expression (figure 2I,J).

\section{E4orf1 protects against the development of hepatic steatosis and prevents fibrosis in mice following long-term HF exposure}

To determine lipid accumulation in the liver, formalinfixed liver sections were $\mathrm{H} \& \mathrm{E}$ stained and pathological microscopic scoring was performed (IDEXX BioResearch). Observed microscopic changes were graded, using the grading system for rodent NAFLD. ${ }^{24}$ The summary scores were calculated for lesions indicative of NAFLD/NASH including macrovesicular steatosis, microvesicular steatosis, hepatocyte hypertrophy, and inflammation. These changes were most extensive in control mice. All mice in the control group had the highest score 3, for macrovesicular and microvesicular lipidosis. Additionally, the control mice had more extensive hepatocellular hypertrophy (due to the microvesicular lipid accumulation filling/expanding the cell; figure $3 \mathrm{~A}, \mathrm{~B})$. While E4orf1 expressing mice had extensive macrovesicular lipidosis, they had less microvesicular and subsequent hypertrophy indicating less severe liver damage. Both groups had inflammation associated with fat accumulation; however, the average inflammation in E4orf1 mice was less than control (figure 3C,D). Fibrosis was also scored on Picro sirius red-stained sections using a standard grading system where $0=$ no significant fibrosis, $1=$ mild fibrosis, $2=$ moderate fibrosis, and $3=$ massive fibrosis. All liver tissues from control mice had significant fibrosis (a common consequence in NAFLD/NASH syndrome) while E4orf1 expressing mice showed mostly normal fibrosis around vasculature fibrosis with a significantly lower fibrosis score (figure $3 \mathrm{E}, \mathrm{G}$ ).

\section{E4orf1 modulates lipid metabolism in the liver}

Attenuation of steatosis and fibrosis in E4orfl mice was further examined by determining changes in hepatic lipid metabolism. We examined the levels of mRNA associated with fatty acid uptake, de novo lipogenesis, fatty acid activation, intracellular fatty acid transport, triglyceride (TG) synthesis, very low density lipoprotein (VLDL) assembly and secretion, and lipid droplet formation in E4orfl expressing transgenic mice compared with control non-transgenic mice as follows:

\section{Fatty acid uptake}

No significant difference was found in the expression of genes associated with fatty acid uptake mRNAs, namely Fabppm, Cd36, Cav-1, Fatp2, Fatp4, Fatp5, and ipla2 for E4orf1 mice compared with control mice (figure 4A). 
A.

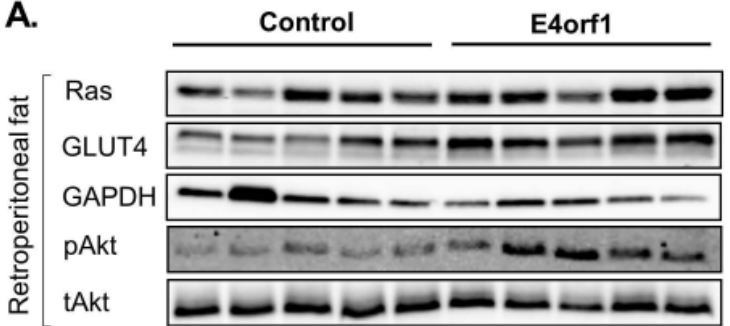

C.

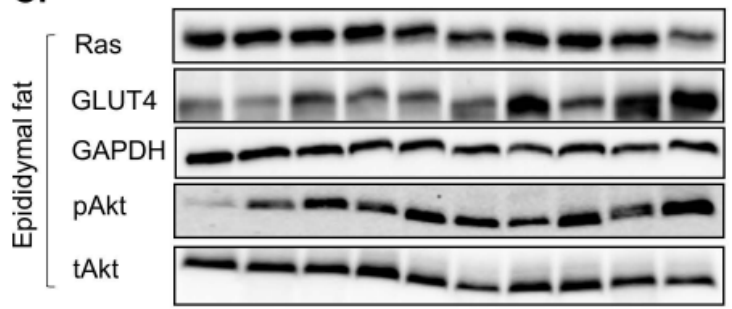

E.

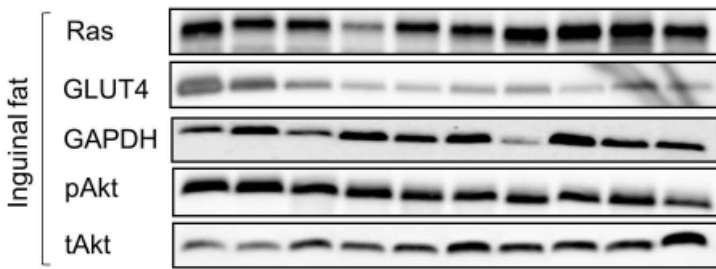

G.

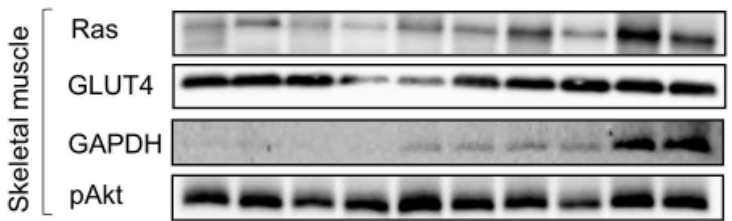

I.

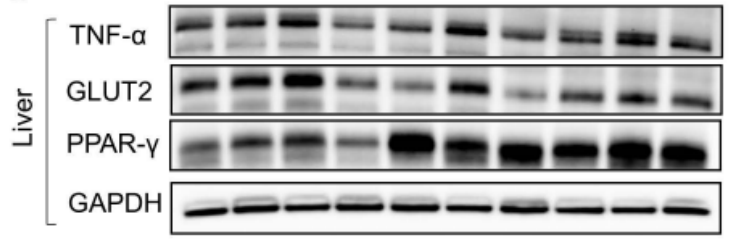

B.

D.
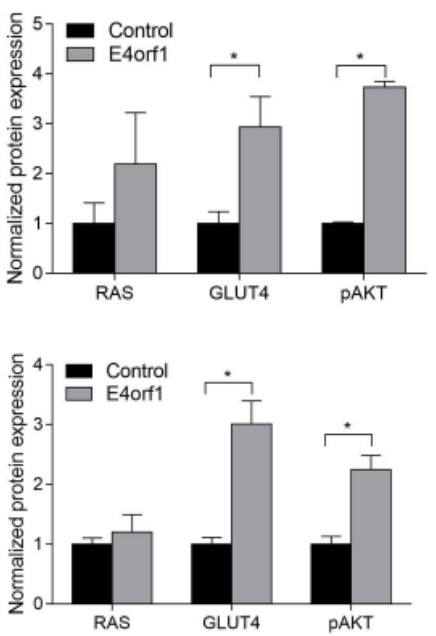

F.

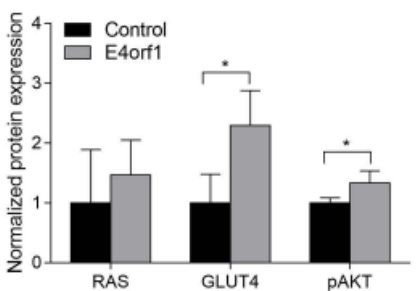

H.

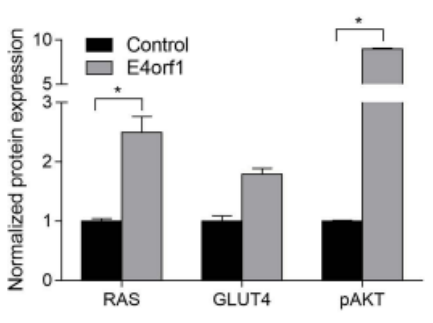

J.

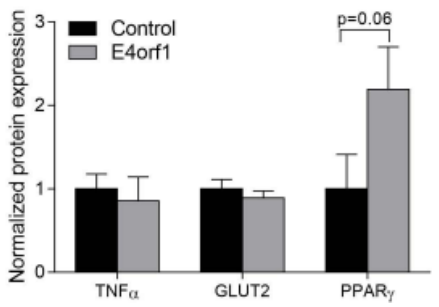

Figure 2 E4orf1 improves glucose metabolism by upregulating proteins of the distal insulin Ras/PI3K signaling pathway in $(A, B)$ retroperitoneal fat depot, $(C, D)$ epididymal fat depot, $(E, F)$ inguinal fat depot, and $(G, H)$ skeletal muscle. But it does not show any difference in $(I, J)$ expression of TNF- $\alpha$, GLUT2, and PPAR- $\gamma$ proteins in the liver. ${ }^{*} \mathrm{p}<0.05$.

\section{De novo lipogenesis}

Among the de novo lipogenesis genes Srebp1c and Fasn, expression was significantly lower in the E4orf1 mice compared with control mice and no difference was observed for Chrebp, Acc1, and $S c d-1$ (figure 4B). We further looked at acyl-coenzymeA molecules involved in thioesterification, catalyzed by various enzymes known as long-chain acyl-coenzymeA synthetases (ACSLs). There was no difference in expression of Acsl genes specifically Acsll, Acsl3, and Acsl5 between E4orfl and control mice (figure 4C). However, among enzymes that catalyze the hydrolysis of acyl-coenzymeA molecules into fatty acid and coenzymeA, known as acyl coenzymeA thioesterases (ACOTs), Acot13
mRNA expression was significantly downregulated in E4orf1 mice compared with control mice (figure 4C).

\section{Intracellular lipid transport}

Intracellular transport and partitioning of long chain fatty acids and acyl-coenzymeAs within hepatocytes are carried out by lipid binding proteins. Among intracellular transport associated genes, $L-f a b p$ was significantly downregulated in E4orf1 mice, but there was no difference for $D b i$ and $S c p-1$ expression (figure 4D).

\section{TG export}

In the liver, Mttp incorporates TG into Apob in the endoplasmic reticulum (ER) followed by additional packaging 


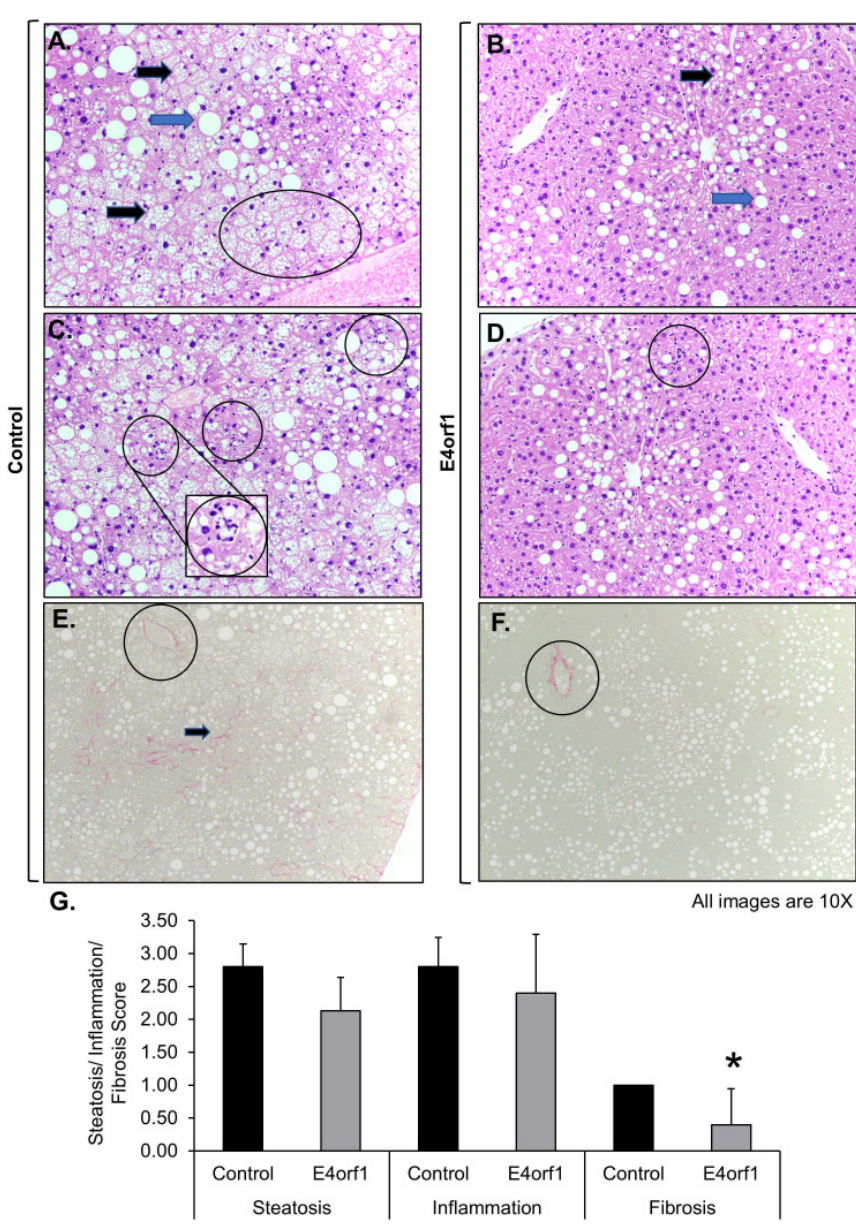

Figure 3 E4orf1 expressing mice decrease hepatic steatosis, inflammation, and protect against fibrosis. H\&E stained liver sections show extensive macrovesicular lipid (blue arrow), microvesicular lipid (black arrow), and hypertrophy (oval marked) in (A) control mice compared with (B) E4orf1 expressing mice. H\&E stained liver sections show presence of more grade 3 inflammation in (C) control mice compared with (D) E4orf1 mice. 10x; insert 20x. Picro sirius red stained liver sections show significant fibrosis (arrows) in (E) control mice compared with normal fibrosis around vasculature (circle) found in (F) E4orf1 expressing mice. (G) Steatosis, inflammation, and fibrosis scores (0-3) graded in liver samples from fat-fed control and E4orf1 expressing mice.

of TG during the translocation from ER to Golgi apparatus and exported as mature VLDL particles. There was no significant difference in mRNA levels for $M t t p$ and $A$ pob between E4orf1 and control mice (figure 4E).

\section{TG synthesis}

The rate limiting step of esterification of long-chain acylcoenzymeA into Glycerol-3-phosphate (G3P) is catalyzed by G3P acyltransferase enzymes (GPATs), which are the major contributor of total TG synthesis in liver. As seen in figure $4 \mathrm{~F}$, the expression of Gpat4 was significantly lower in E4orf1 mice compared with control mice, while there was no significant difference for Gpat1, Gpat2, and Gpat3 (figure 4F). Similarly, the expression of enzymes that catalyze the acylation of diacylglycerol in the final step of
TG synthesis, Dgat1 and Dgat2, was not different between E4orf1 and control mice (figure 4F). Also, there was no difference in the expression of Lipin-1, another contributor of the TG biosynthetic pathway (figure 4F).

\section{Fat oxidation}

To determine fat oxidation in the liver, we measured the

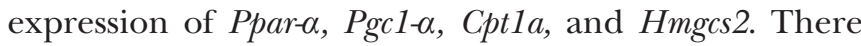
was no significant difference for the fat oxidation genes between E4orf1 and control mice (figure 4G). Lipid droplets (LDs) play an important role in hepatic lipid storage by transiently storing lipids in the liver. The LD-associated proteins are involved in the formation, expansion, and contraction; however, we did not observe any difference in expression among mRNA levels of perilipin and the DFF45-like effector/CIDE in E4orf1 or control mice (figure $4 \mathrm{H}$ ). To determine changes in glucose and insulin metabolism and NAFLD, we also examined Foxo1, Hsd11/1, Fgf21, G6pase, and Pepck. None of these genes showed any significant difference in expression between E4orf1 and control mice (figure 4I,J). Although the histological analyses suggest reduced hepatic fibrosis (figure 3E), none of the genes associated with fibrogenesis were significantly different (Col1a, Col3a, Acta2, $T g f \beta 1$, and $C \operatorname{tgf})$ between E4orf1 and control mice (figure 4K).

\section{E4orf1 protects mitochondrial integrity and reduces the indicators of cellular aging in mice}

To explore the effect of E4orfl on aging-related pathways of HFD-fed older mice, we determined the expression of aging-related genes using $\mathrm{RT}^{2}$ profiler PCR array (figure 5). Out of 84 genes, 24 were found differentially expressed $(\mathrm{p}<0.05)$ (figure 6). STRING analyses indicate the differential expression of genes are associated with mitochondrial biogenesis and integrity (Tfam, $T f b 1 m, T f b 2 m$, and Sirt3), telomere stabilizing shelterincomplex (Terf2 and Tpp1), and protection of the liver (Cd163, Tollip, and Clu) in E4orf1 mice compared with the control group. Also, $C \times 3 c l 1$ (fractalkine) gene, which is implicated in anti-inflammatory response, was significantly elevated in E4orf1 mice.

\section{DISCUSSION}

Aging is an independent risk factor for many chronic diseases $^{1}$ and advanced age leads to deterioration in many key cellular and tissue dysfunction, which together contribute to generate metabolic disorders. ${ }^{2}$ Even though aging is inevitable, modifying chronic diseases that either manifest with age or exacerbate aging associated risk factors can facilitate improved health span and potentially life span.

In older adults, T2D is one of the most common metabolic diseases and several factors contribute in the pathophysiology of T2D in older age. NAFLD is the most common liver disease worldwide, affecting $\sim 24 \%$ of the world's population. ${ }^{25}$ NAFLD encompasses a wide range of pathological conditions ${ }^{26}$ and 
A.

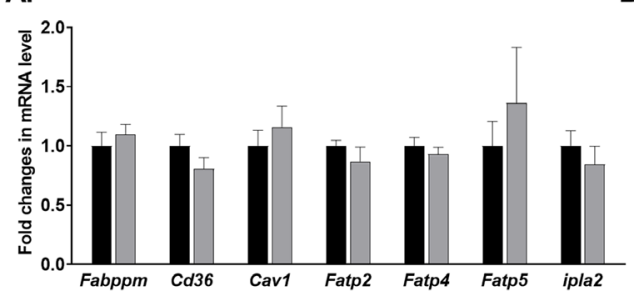

C.

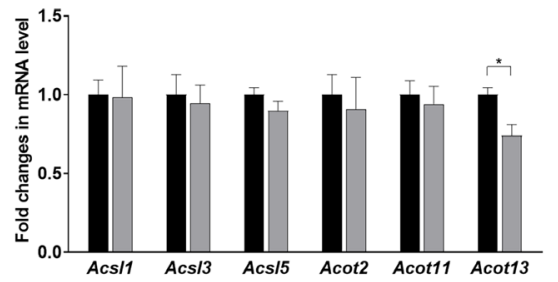

F.

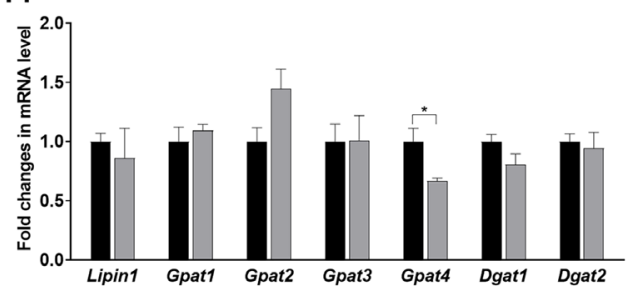

H.

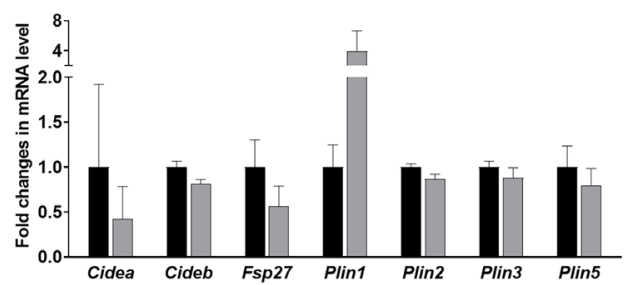

B.

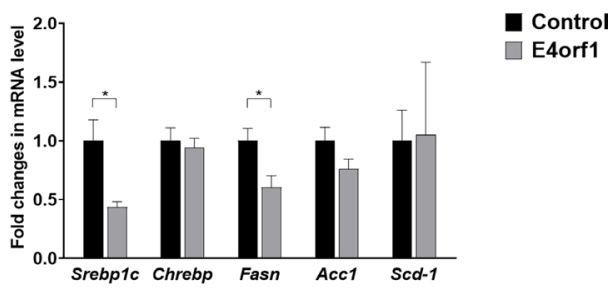

D.

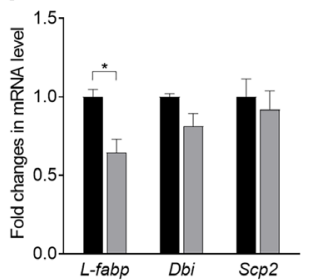

E.

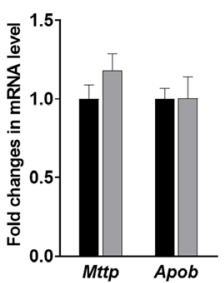

G.

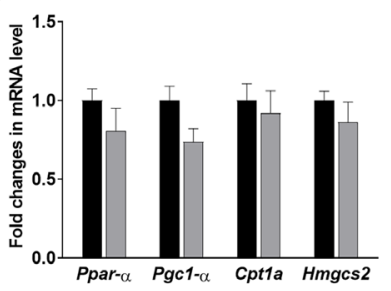

I.

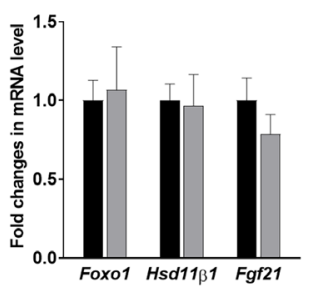

J.

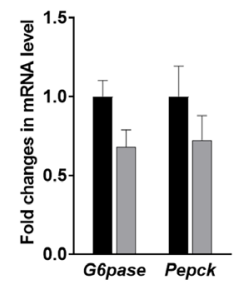

K.

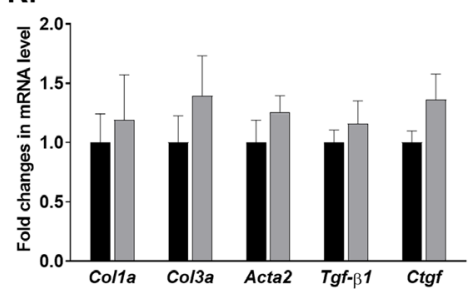

Figure 4 No changes in expression of hepatic lipid metabolism-related genes. Expression of genes associated with (A) fatty acid uptake, (B) de novo lipogenesis, (C) VLDL assembly and secretion, (D) intracellular fat transport-associated genes, (E) TG export, (F) TG synthesis, (G) fat oxidation and fatty acid activation, (H) lipid droplet biology, (I) expression of Foxo1, Hsd11 $\beta 1$, and Fgf21 genes, (J) expression of G6pase and Pepck genes, and (K) fibrosis. Welch's $t$-test: *p<0.05.TG, triglyceride; VLDL, very low density lipoprotein.

the risk of NAFLD is twofold higher in individuals with T2D, which could progress to steatohepatitis and fibrosis. ${ }^{27}$ NAFLD is common among the elderly and carries a more substantial burden of hepatic (NASH, cirrhosis and hepatocellular carcinoma) and extrahepatic manifestations and complications (cardiovascular disease, extrahepatic neoplasms) compared with younger age groups. ${ }^{28}$ Collectively, these metabolic disorders affect factors of aging and therefore, proper identification and successful management of these intrinsic metabolic changes should provide an effective approach for improving healthy longevity.
The E4orf1 protein, derived from human adenovirus Ad36, improves glucose clearance in an insulin independent manner despite presence of HFD in mice. ${ }^{17-19} 29$ In cells, E4orf1 bypasses the proximal insulin signaling (insulin receptor or insulin receptor substrate IRS-1/2, but upregulates distal insulin signaling (Ras-PI3K-AKT) to increase cellular glucose uptake, ${ }^{30}$ even in the presence of inflammation. ${ }^{31}$ Therefore, E4orf1 might have a therapeutic role in improving glycemic control under conditions of insulin resistance or impaired insulin signaling and inflammation, which are often observed during aging. 


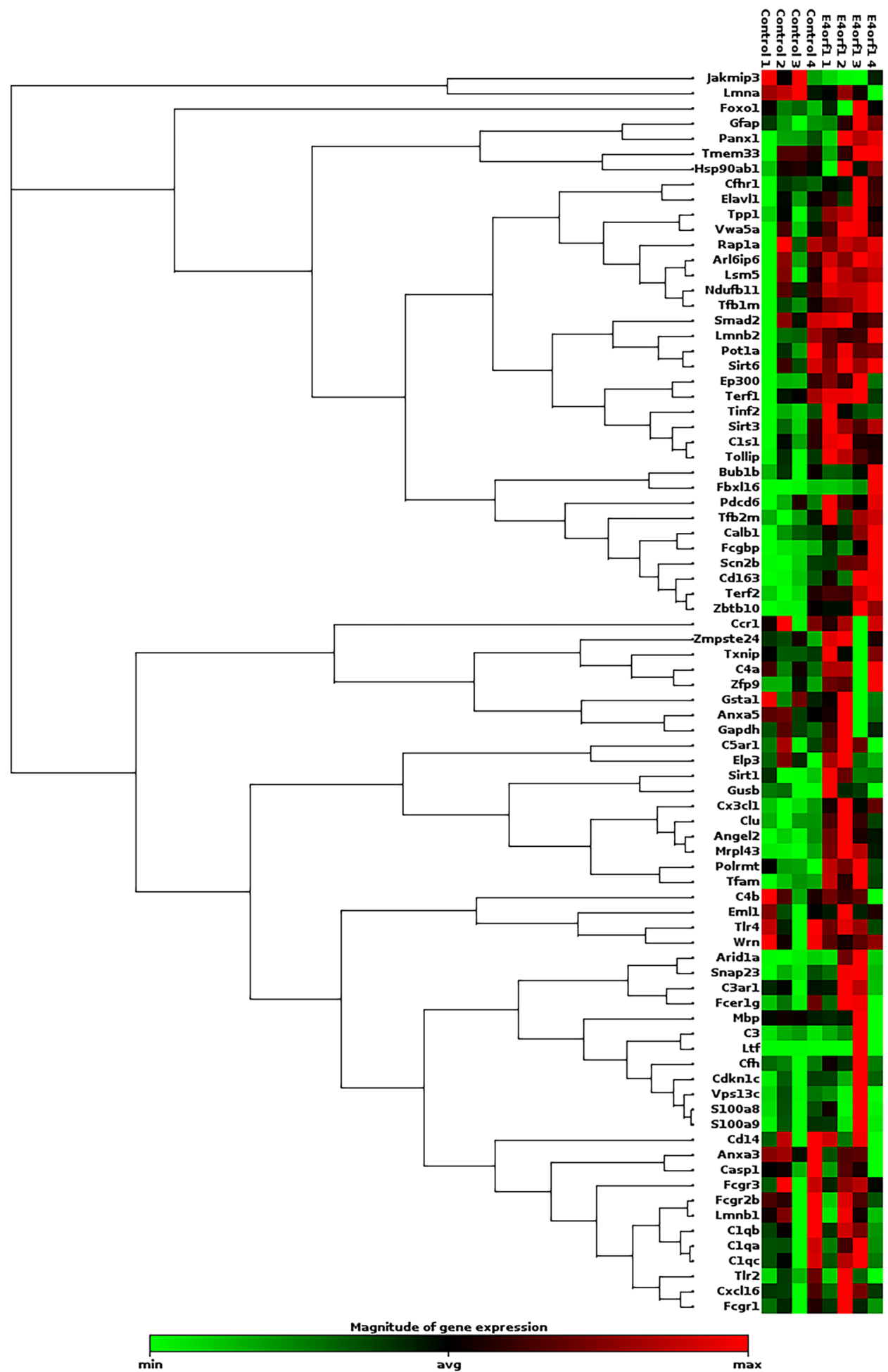

Figure 5 Heat map of the $\mathrm{RT}^{2}$ aging PCR array: age and HFD induced changes in gene expression in the liver of the control and E4orf1 group. Expression of aging-related genes of each mouse is listed in columns and grouped in control and E4orf1. The genes are listed in the rows and color changes from green to red show increased expression in fold changes. HFD, high fat diet.

Adipose-tissue specific expression of E4orf1 reduces the requirement endogenous insulin and provides protection against hepatic steatosis in HFD-fed mice. ${ }^{17}$ Hepatic steatosis or NAFLD has components of the metabolic syndrome and increasing age seems to have an influence in increasing the prevalence of NAFLD. Advanced age is associated with disease severity and progression to NASH, cirrhosis and liver failure, where fibrosis during NASH is the most robust determinant of all cause and liver related mortality. ${ }^{32}$ In the current study, E4orfl expression improved expression of genes involved in hepatic fatty acid 


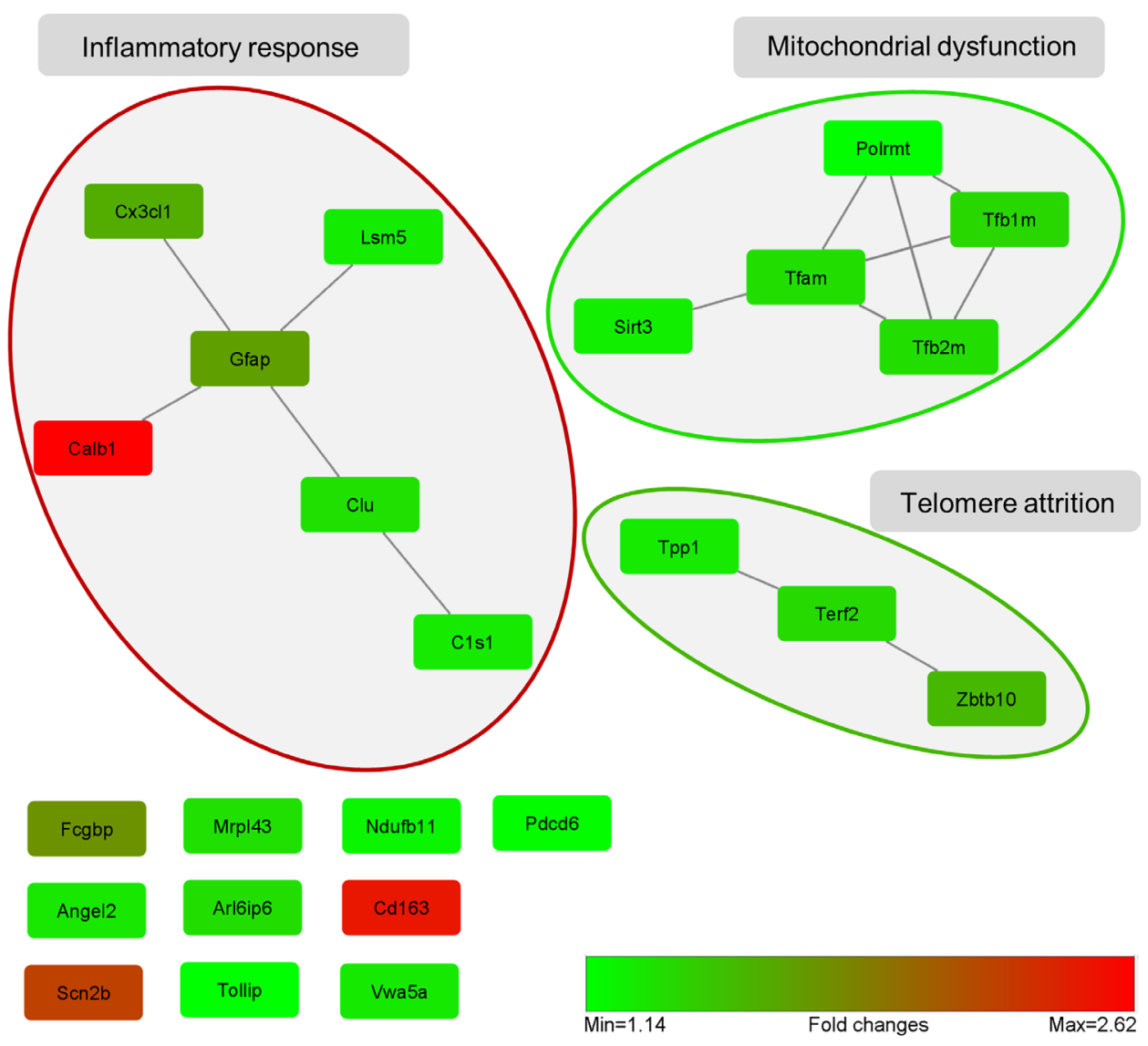

Figure 6 Gene set network of liver-aging $\mathrm{RT}^{2} \mathrm{PCR}$ array analysis of the E4orf1 expressing group compared with the control group. Out of 24 significantly differentially expressed genes, 14 were linked with inflammatory response, mitochondrial dysfunction, and telomere attrition.

metabolism and liver histology showed that E4orf1 expression significantly ameliorated development of fibrosis in older mice exposed to chronic HFD, which suggests attenuation of hepatic cellular deterioration and in turn risk factors of aging. To further explore E4orf1 mediated changes in risk factors of aging, we determined expression of aging related genes in the liver from older mice on a $60 \%$ HFD for 20 weeks.

Our RT ${ }^{2}$ profiler PCR array data show that Tfam, $T f b 1 m$, and $T f b 2 m$ gene expression were significantly higher in the liver of 15-month-old E4orf1 mice compared with control group, suggesting possible protection of mitochondrial integrity in E4orf1 mice. Aging is influenced by reduced production of ATP resulting from age-dependent mitochondrial dysfunction, ${ }^{33}$ and concomitant reduction of mitochondrial biogenesis, which are common to several agingassociated diseases such as T2D and Alzheimer's disease $(\mathrm{AD}){ }^{34}$ Therefore, mitochondrial biogenesis becomes critical for mitochondrial homeostasis to meet the physiological need of cells. Mitochondrial biogenesis initiation can occur via the PGC-1 family of proteins, leading to activation of mitochondrial transcription factors A (TFAM), B1 (TFB1M), and B2 (TFB2M). ${ }^{35}$ TFAM, TFB1M, and TFB2M are involved in various cellular functions; specifically, TFAM is pivotal to mitochondrial biogenesis with its role in mitochondrial-DNA (mtDNA) transcription, maintenance, repair, and replication. ${ }^{35}$ The disruption of Tfam in mice exhibits reduced mtDNA, respiratory deficiency, and abolished oxidative phosphorylation. ${ }^{36}$ Although a detailed mechanism is yet to be determined, elevated expression of Tfam, $T f b 1 \mathrm{~m}$, and $T f b 2 m$ in HFD-fed E4orf1 mice could be due to improved glycemic control. Hyperglycemia-induced ROS production decreases mitochondrial function measured by reduced mtDNA and Tfam level in HepG2 cells. ${ }^{37}$ Furthermore, E4orf1 upregulates the mRNA expression of PGC- $1 \alpha$ in liver. ${ }^{17}$ PGG- $1 \alpha$ is a member of the PGC-1 family, known to initiate the mitochondrial biogenesis pathway. In addition to the mitochondrial transcription factors, Sirtuin 3 (Sirt3) mRNA level was significantly higher in the liver of E4orf1 mice. Sirt3 is a nicotinamide adenine dinucleotide (NAD)-dependent deacetylase, which provides mitochondrial protection in response to oxidative stress, hyperglycemia, and fatty acid exposure. ${ }^{38}$ Sirt3 activation promotes acylcarnitine metabolism and protects against NAFLD,${ }^{28}$ which correlates with liver histology observed with E4orf1 mice. We 
also found genes for two members of the shelterincomplex, Terf2 and Tpp1 upregulated in the liver of E4orf1 mice. Shelterin-complex, a group of six proteins (TERF1, TERF2, POT1, RAP1, TIN2, and TPP1) stabilize telomers and prevent the unyielding loss of telomeres implicated in the longevity of various species. ${ }^{39}$ TERF2 plays a vital role in telomere maintenance and prevents fusion of chromosomal ends by binding to the telomeric double-stranded 5'-TTAGGG-3' repeat. ${ }^{40}$ Intriguingly, according to a recent finding, Terf 2 can activate Sirt 3 and consequently control mitochondrial function in cultured human myotubes and mouse skeletal muscle. ${ }^{30}$ Even though our findings are from the liver, these observations warrant a more detailed investigation. TPP1 is another key member of the shelterin-complex and forms heterodimer with POT1, TPP1-POT1, which on binding to TIN2 interacts with TERF1 and TERF2 to ally with telomers. ${ }^{41}$ Zbtb10 is affiliated with TERF2 and involved in alternative lengthening of telomeres (ALT), ${ }^{42}$ which has a higher expression in E4orf1 mice. Among other upregulated mRNAs in the liver of E4orf1 mice, Cd163, Tollip, and Clu are implicated in the protection against liver steatosis, inflammation, and $\mathrm{NASH},{ }^{43}{ }^{44}$ which is in agreement with the observed gene and liver histology data with E4orf1 mice. Cx3cl1 exhibits anti-inflammatory feature by increasing the expression of Il-10 and arginase-1 in Kupffer cells and can inhibit liver inflammation and fibrosis. ${ }^{45}$ CX3CL1 is associated with cell recruitment and cell survival through binding to CX3C-chemokine receptor 1 (CX3CR1), and AKT and ERK pathway. ${ }^{45}$ In contrast, Glial fibrillar acidic $(G f a p)$ protein and Clusterin 1 are found elevated, which are implicated in liver diseases. However, the function of Arl6ip6, Angel2, C1s1, Pdcd6, Vwa5a, and Lsm5 is not well described in the literature.

In conclusion, these results suggest that the insulinindependent action of E4orf1 reduces endogenous insulin secretion and improves glycemic control despite HFD in mice. Reduced insulin secretion likely protects the liver against lipid accumulation, which prevents NAFLD and its progression to fibrosis and NASH. Improvement in these metabolic features may protect against ageassociated cellular aging by maintaining mitochondrial integrity and telomere attrition. These observations have significant implications in humans for development of E4orf1 as a therapeutic target. Excess nutrient intake causes obesity and shares several hallmarks of aging ${ }^{46}$ affecting cellular processes and accelerating aging. ${ }^{47}$ We recently reported nanoparticle-mediated delivery of E4orf1 as a clinically relevant delivery system. ${ }^{48}$ Therefore, the ability of E4orf1 in improving glycemic control, protecting against hepatic fat accumulation and reducing aging-associated cellular damage despite chronic HFD will be clinically desirable. Furthermore, in humans, chronic consumption of HFDs is associated with increased risk of cognitive decline and dementia ${ }^{49}$ and in a mouse model of $\mathrm{AD}$, we have shown that Ad36 improves glycemic control and ameliorates cognitive decline ${ }^{50}$ Collectively, these observations underscore the need for detailed studies for development of E4orfl as a therapeutic intervention to improve glycemic control and prevention of age-associated diseases.

Contributors ZM researched data and reviewed/edited manuscript. MA wrote the manuscript and researched data. MABS researched data and reviewed/edited the manuscript. PP researched data. NVD contributed to discussion and reviewed/ edited the manuscript. VH researched data and wrote the manuscript.

Funding This work was supported in part by startup funds from Texas Tech University, College of Human Sciences and Department of Nutritional Sciences and by American Heart Association AIREA grant (20AIREA35170031) to VH.

Competing interests NVD has received several US and international patents that protect intellectual property about the use of adenoviruses and its proteins in obesity, diabetes, and related areas.

Patient consent for publication Not required.

Provenance and peer review Not commissioned; externally peer reviewed.

Data availability statement Data are available on reasonable request. All data relevant to the study are included in the article.

Open access This is an open access article distributed in accordance with the Creative Commons Attribution Non Commercial (CC BY-NC 4.0) license, which permits others to distribute, remix, adapt, build upon this work non-commercially, and license their derivative works on different terms, provided the original work is properly cited, appropriate credit is given, any changes made indicated, and the use is non-commercial. See: http://creativecommons.org/licenses/by-nc/4.0/.

ORCID IDs

Md Akheruzzaman http://orcid.org/0000-0003-4639-5581

Md Abu Bakkar Siddik http://orcid.org/0000-0002-3550-946X

Vijay Hegde http://orcid.org/0000-0003-4160-2764

\section{REFERENCES}

1 Barzilai N, Cuervo AM, Austad S. Aging as a biological target for prevention and therapy. JAMA 2018;320:1321-2.

2 Tchkonia T, Kirkland JL. Aging, cell senescence, and chronic disease: emerging therapeutic strategies. JAMA 2018;320:1319-20.

3 Slawik M, Vidal-Puig AJ, Lipotoxicity V-PAJ. Lipotoxicity, overnutrition and energy metabolism in aging. Ageing Res Rev 2006;5:144-64.

4 Barzilai N, Huffman DM, Muzumdar RH, et al. The critical role of metabolic pathways in aging. Diabetes 2012;61:1315-22.

5 Blackburn EH. Structure and function of telomeres. Nature 1991;350:569-73.

6 Tamura Y, Izumiyama-Shimomura N, Kimbara Y, et al. $\beta$-cell telomere attrition in diabetes: inverse correlation between $\mathrm{HbA1c}$ and telomere length. J Clin Endocrinol Metab 2014;99:2771-7.

7 Zee RYL, Castonguay AJ, Barton NS, et al. Mean leukocyte telomere length shortening and type 2 diabetes mellitus: a case-control study. Trans/ Res 2010;155:166-9.

8 Balaban RS, Nemoto S, Mitochondria FT. Oxidants, and aging. Cell 2005;120:483-95.

9 Sahin E, Colla S, Liesa M, et al. Telomere dysfunction induces metabolic and mitochondrial compromise. Nature 2011;470:359-65.

10 Dassanayaka S, Readnower RD, Salabei JK, et al. High glucose induces mitochondrial dysfunction independently of protein OGlcNAcylation. Biochem J 2015;467:115-26.

11 Xia X, Chen W, McDermott J, et al. Molecular and phenotypic biomarkers of aging. F1000Res 2017;6:860.

12 Omodei D, Fontana L. Calorie restriction and prevention of ageassociated chronic disease. FEBS Lett 2011;585:1537-42.

13 Mercken EM, Carboneau BA, Krzysik-Walker SM, et al. Of mice and men: the benefits of caloric restriction, exercise, and mimetics. Ageing Res Rev 2012;11:390-8.

14 Brewer RA, Gibbs VK, Smith DL. Targeting glucose metabolism for healthy aging. Nutr Healthy Aging 2016;4:31-46.

15 De Haes W, Frooninckx L, Van Assche R, et al. Metformin promotes lifespan through mitohormesis via the peroxiredoxin PRDX-2. Proc Natl Acad Sci U S A 2014;111:E2501-9. 
16 Bannister CA, Holden SE, Jenkins-Jones S, et al. Can people with type 2 diabetes live longer than those without? A comparison of mortality in people initiated with metformin or sulphonylurea monotherapy and matched, non-diabetic controls. Diabetes Obes Metab 2014;16:1165-73.

17 Akheruzzaman M, Hegde V, Shin AC, et al. Reducing endogenous insulin is linked with protection against hepatic steatosis in mice. Nutr Diabetes 2020;10:11.

18 Kusminski CM, Gallardo-Montejano VI, Wang ZV, Hegde V, et al. E4orf1 induction in adipose tissue promotes insulin-independent signaling in the adipocyte. Mol Metab 2015;4:653-64.

19 McMurphy TB, Huang W, Xiao R, et al. Hepatic expression of adenovirus 36 E4ORF1 improves glycemic control and promotes glucose metabolism through Akt activation. Diabetes 2017;66:358-71.

20 Pasarica M, Shin AC, Yu M, et al. Human adenovirus 36 induces adiposity, increases insulin sensitivity, and alters hypothalamic monoamines in rats. Obesity 2006;14:1905-13.

21 Krishnapuram R, Dhurandhar EJ, Dubuisson O, et al. Template to improve glycemic control without reducing adiposity or dietary fat. Am J Physiol Endocrinol Metab 2011;300:E779-89.

22 Dhurandhar NV. A framework for identification of infections that contribute to human obesity. Lancet Infect Dis 2011;11:963-9.

23 Trovato GM, Martines GF, Garozzo A, et al. Ad36 adipogenic adenovirus in human non-alcoholic fatty liver disease. Liver Int 2010;30:184-90.

24 Liang W, Menke AL, Driessen A, et al. Establishment of a general NAFLD scoring system for rodent models and comparison to human liver pathology. PLoS One 2014;9:e115922.

25 Bedogni G, Miglioli L, Masutti F, et al. Prevalence of and risk factors for nonalcoholic fatty liver disease: the Dionysos nutrition and liver study. Hepatology 2005;42:44-52.

26 Yeh MM, Brunt EM. Pathological features of fatty liver disease. Gastroenterology 2014;147:754-64.

27 Bril F, Cusi K. Nonalcoholic fatty liver disease: the new complication of type 2 diabetes mellitus. Endocrinol Metab Clin North Am 2016;45:765-81.

28 Wang G-E, Li Y-F, Zhai Y-J, et al. Theacrine protects against nonalcoholic fatty liver disease by regulating acylcarnitine metabolism. Metabolism 2018;85:227-39.

$29 \mathrm{Na} \mathrm{H}-\mathrm{N}$, Hegde V, Dubuisson O, et al. E4orf1 enhances glucose uptake independent of proximal insulin signaling. PLoS One 2016;11:e0161275.

30 Robin JD, Jacome Burbano M-S, Peng H, et al. Mitochondrial function in skeletal myofibers is controlled by a TRF2-SIRT3 axis over lifetime. Aging Cell 2020;19:e13097.

$31 \mathrm{Na} \mathrm{H}-\mathrm{N}$, Dubuisson O, Hegde V, et al. Human adenovirus Ad36 and its E4orf1 gene enhance cellular glucose uptake even in the presence of inflammatory cytokines. Biochimie 2016;124:3-10.

32 Vilar-Gomez E, Calzadilla-Bertot L, Wai-Sun Wong V, et al. Fibrosis severity as a determinant of cause-specific mortality in patients with advanced nonalcoholic fatty liver disease: a Multi-National cohort study. Gastroenterology 2018;155:443-57.
33 Navarro A, Boveris A. Rat brain and liver mitochondria develop oxidative stress and lose enzymatic activities on aging. Am J Physiol Regul Integr Comp Physiol 2004;287:R1244-9.

34 Jornayvaz FR, Shulman GI, Brown GC. Regulation of mitochondrial biogenesis. Essays Biochem 2010;47:69-84.

35 Picca A, Lezza AMS. Regulation of mitochondrial biogenesis through TFAM-mitochondrial DNA interactions: useful insights from aging and calorie restriction studies. Mitochondrion 2015;25:67-75.

36 Larsson NG, Wang J, Wilhelmsson $\mathrm{H}$, et al. Mitochondrial transcription factor $A$ is necessary for mtDNA maintenance and embryogenesis in mice. Nat Genet 1998;18:231-6.

37 Palmeira CM, Rolo AP, Berthiaume J, et al. Hyperglycemia decreases mitochondrial function: the regulatory role of mitochondrial biogenesis. Toxicol Appl Pharmacol 2007;225:214-20.

38 Papavagelis C, Avgeraki E, Augoulea A, et al. Dietary patterns, Mediterranean diet and obesity in postmenopausal women. Maturitas 2018;110:79-85.

39 Srinivas N, Rachakonda S, Kumar R. Telomeres and telomere length: a general overview. Cancers 2020;12. doi:10.3390/ cancers12030558. [Epub ahead of print: 28 Feb 2020].

40 Karlseder J, Broccoli D, Dai Y, et al. P53- and ATM-dependent apoptosis induced by telomeres lacking TRF2. Science 1999;283:1321-5.

41 Frescas D, de Lange T. Binding of TPP1 protein to TIN2 protein is required for POT1a,b protein-mediated telomere protection. J Biol Chem 2014;289:24180-7.

42 Bluhm A, Viceconte N, Li F, et al. ZBTB10 binds the telomeric variant repeat TTGGGG and interacts with TRF2. Nucleic Acids Res 2019;47:1896-907.

43 Park J-S, Shim Y-J, Kang B-H, et al. Hepatocyte-Specific clusterin overexpression attenuates diet-induced nonalcoholic steatohepatitis. Biochem Biophys Res Commun 2018;495:1775-81.

44 Chen K, Yuan R, Zhang Y, et al. Tollip deficiency alters atherosclerosis and steatosis by disrupting lipophagy. J Am Heart Assoc 2017;6. doi:10.1161/JAHA.116.004078. [Epub ahead of print: 10 Apr 2017].

45 Aoyama T, Inokuchi S, Brenner DA, et al. Cx3Cl1-Cx3Cr1 interaction prevents carbon tetrachloride-induced liver inflammation and fibrosis in mice. Hepatology 2010;52:1390-400.

46 López-Otín C, Blasco MA, Partridge L, et al. The hallmarks of aging. Cell 2013;153:1194-217.

47 Pérez LM, Pareja-Galeano H, Sanchis-Gomar F, et al. 'Adipaging': ageing and obesity share biological hallmarks related to a dysfunctional adipose tissue. J Physiol 2016;594:3187-207.

48 Feizy Z, Peddibhotla S, Khan S, et al. Nanoparticle-mediated in vitro delivery of E4orf1 to preadipocytes is a clinically relevant delivery system to improve glucose uptake. Int J Obes 2020;44:1607-16.

49 Dye L, Boyle NB, Champ C, et al. The relationship between obesity and cognitive health and decline. Proc Nutr Soc 2017;76:443-54.

50 Hegde V, Vijayan M, Kumar S, et al. Adenovirus 36 improves glycemic control and markers of Alzheimer's disease pathogenesis. Biochim Biophys Acta Mol Basis Dis 1865;2019:165531. 